Research Articles

\title{
Preparation of Magnetorheological Fluids Using Different Carriers and Detailed Study on Their Properties
}

\author{
${ }^{1,2}$ Vikram G. Kamble, ${ }^{2}$ Shreedhar Kolekar and ${ }^{2,3}$ Chetan Madivalar \\ ${ }^{1}$ Proof and Experimental Establishment (DRDO), Balasore, Odisha-756025, India \\ ${ }^{2}$ St Joseph Engineering College, Mangalore, Karnataka-575028, India \\ ${ }^{3}$ Department of Mechanical Engineering, Eastern Michigan University, Ypsilanti, Michigan, USA
}

Article history

Received: 20-06-2015

Revised: $16-07-2015$

Accepted: 21-08-2015

Corresponding Author:

Vikram G Kamble

Proof and Experimental

Establishment (DRDO),

Balasore, Odisha-756025, India

Tel: +91 9901447793

E-mail: iknowvikramkamble@gmail.com

\begin{abstract}
High sensitive and intelligence are the main function of the Magneto Rheological Fluids (MRF). In this research work, magneto rheological samples are prepared with two different carrier oils (Honge and Castor) independently. Both the samples are mixed and prepared with same concentration of iron particles. In order to reduce the sedimentation fine lithium grease is added as stabilizer for both samples. The flow properties are examined by cup and bob type rheometer. Then comparative study is made on the properties like shear stress, strain, shear rate, dynamic viscosity, angular frequency, storage modulus, loss modulus and loss factor.
\end{abstract}

Keywords: Strain, Dynamic Viscosity, Angular Frequency, Stress, Shear Rate, Storage Modulus, Loss Modulus, Loss Factor

\section{Introduction}

The term smart material generally refers to the material which changes its properties under the influence of various external stimuli (Garg and Anderson, 2000). However, it seems that this approach is too easy. Much more precise description of smart material is given by Takagi in his work (Takagi, 1999). According to him, smart material is capable of reacting to external stimuli. Such material should therefore be some kind of sensors, processors and actuators (Boczkowska and Awietjan, 2012). Till today there is no accepted universal definition of smart materials, it is also not included in the encyclopedia devoted to these materials, published in 2002 (Schwartz, 2002). The word "magneto rheological" is used only in relation to a group of magneto rheological materials. It describes the reversible properties changes under the magnetic field in comparison to the material properties without the presence of the field. Changes in the magneto rheological properties are usually described by the shear modules: storage modulus $\left(\mathrm{G}^{\prime}\right)$ and loss modulus $\left(\mathrm{G}^{\prime \prime}\right)$ (Carlson and Jolly, 2000; Lokander et al., 2004; Borcea and Bruno, 2001; Demchuk and Kuzmin, 2002; Yalcintas and Dai, 2004; Zhou and Jiang, 2004; Dorfmann and Ogden, 2003).

The study of flow and deformation of fluids in which solid particles are dispersed is called as rheology. The type of fluids can be sludge, suspensions, mud, polymers or bodily fluids. The magneto-rheological fluids (MR fluids) contain magnetizable particles, when these particles are dispersed in synthetic fluids they show a characteristic change in the properties that are rapid and reversible in nature (Ray et al., 2002). This reversible nature of MR fluids has many applications in automobile suspensions and earthquake mitigation structures in which the amount of damping can be variably controlled by varying the intensity of magnetic field (Chiriac and Stoian, 2010).

The MR fluids basically consist of carrier oil, a binder and the magnetizable particles. The carrier fluid is mixed with the binder along with the iron particles and stirred for long intervals, for proper dispersion and uniform concentration. The carrier fluid constitutes for a $60 \%$ to $80 \%$ of the volume in MR fluid, the ratio can be varied according to the requirement. The binder constitutes for $10 \%$ to $20 \%$ of the volume in an MR fluid and the rest of the volume is occupied by iron particles that can be magnetized (Ginder et al., 2002; Böse and Röder, 2009; Zhou, 2003).

When a magnetic field is applied to the MR fluid, alignment of the particles takes place. This occurs due to external action of magnetic field but after removal of magnetic field, the particles re-align themselves and come back to the natural state, i.e., the particles get suspended after removal of magnetic field. The amount by which the particles get suspended depends upon the type of carrier fluid used (Boczkowska and Awietjan, 2011; Ginder et al., 2002). The types of carrier fluids used are kerosene, castor oil, honge oil, synthetic oils, polyvinyl-n-butyl, silicon oil, water-based oils. Naturally 
available oils like honge oil and castor oil are cheaper as compared to hydrocarbon oils like silicon oil (Park et al., 2001). The stability of the MR fluid is a very important factor, i.e., the sedimentation problem has to be considered while selecting an MR fluid. Even more important than sedimentation is the durability of MR fluid. If an MR fluid is thinned on shearing, the properties of MR fluid are lost (Kankanala and Triantafyllidis, 2004; Zhou, 2003). Therefore the rate of sedimentation along with good durability has to be there for selecting a good MR fluid. The ionic fluids are very stable in nature, as they do not have a phase separation of the particles as compared to other MR fluids. The ionic fluids are not as durable as conventional MR fluids. The rheometer that we use for measuring the shear rate and shear stress is the cone and plate type rheometer. The fluid is placed on a very shallow plate and a cone is placed on the plate. There is a shaft, which rotates inside the cone, the degree of twist of the shaft gives the shear stress and the rotational speed and the dimensions of the cone gives the shear rate.The degree of change is related to the magnitude of the applied magnetic field and can cover in only few milliseconds (Kamble and Kolekar, 2014).

The main aim of this work is to study the properties of MR fluids and rate of sedimentation of the micron sized iron particles dispersed in the carrier fluid and to comment on their stability.

\section{Preparation of MR Fluid Samples}

Two samples of MR fluids are prepared with carbonyliron particles with particle size $2 \mu \mathrm{m}$ to $5 \mu \mathrm{m}$ and density $7830 \mathrm{~kg} / \mathrm{m}^{3}$. The samples are prepared by taking the required quantity of carrier oil and carbonyl-iron particles.

The surfactant used was fine white Lithium grease, the grease was thoroughly mixed with carrier oil for around 2 hours and then the carbonyl-iron particles were added to the mixture and were well stirred for another one hour. Hence, we could obtain a homogenous mixture of MR fluid. Then it is tested in the cup and bob type rheometer. Preparation of magneto-rheological fluid is explained in article (Kolekar et al., 2014; Kamble and Rvadi, 2015).

In this work, we are using the percentage of elements by weight method, since it involves a mixture of solid and liquid particles and it is difficult to do it by elements by volume method. Table 1 shows the composition of the prepared samples.

\section{Material Properties}

\section{Lithium Grease}

Grease is a semi-solid, which contains lubricating oil, which could be either mineral oil or vegetable oil. The grease possesses a feature by which it has high initial viscosity and on application of shear force the viscosity drops and it gives the same effect of oil. This is the thixotropic nature of grease. Table 2 shows the Properties of the Lithium Grease.

\section{Carbonyl Iron Particles}

The iron powder particles are easily magnetizable and are spherical in shape. The average particle size of these grains was approximately $4-9 \mu \mathrm{m}$ as BASF norms. Along with that, iron powder particle's purity was as high as $99.5 \%$.

\section{Honge and Castor Oils}

Both the oils are synthetic oils and perform the following properties. Table 3 shows the Properties of the both Castor and Honge Oil.

\section{Test for Sedimentation and Stability of MR Fluid}

Sedimentation is a process by which the denser particles tend to settle down in the fluid in which they are present as a mixture and come down to rest as a result of gravity. The other reasons of sedimentation may be centrifugal force or electromagnetic force, which can capture the particles away from the turbid fluid leaving the fluid to be supernatant. The rate of sedimentation is determined by neglecting the magnetic forces and the effect of magnetic field on the MR particles. The density difference between the carrier oil and the iron particles causes the sedimentation of the iron particles, these iron particles tend to settle down and form a dense turbid part at the bottom and leaving clear oil at the top which is called as the supernatant layer. There forms a line separating the supernatant layer and the turbid dense part. By observing the ratio of change in the distance between the two layers with respect to time sedimentation ratio can be determined (Mangal et al., 2013).

\section{Experimental Setup for Sedimentation}

The prepared MR fluids are put into four test tubes, each filled with $10 \mathrm{~cm}$ of MR fluid. The sedimentation test is done visually, hence it is better to place the setup indoors and check the clear view of separation of turbid and supernatant layer when proper illumination is available. The length of clear fluid separating from the dense turbid MR fluid is noted down at regular intervals of time. The Fig. 1 shows the experimental set up of sedimentation test.

\section{Results and Discussion}

Figure $2 \mathrm{a}$ and $\mathrm{b}$ shows the sedimentation ratio of iron particles with grease as additive. 
Table 1.Composition of samples weight percentage of materials with respect to fluid weight

\begin{tabular}{|c|c|c|c|c|c|}
\hline \multicolumn{3}{|c|}{ Honge } & \multicolumn{3}{|c|}{ Castor } \\
\hline $\mathrm{Fe}$ & Grease & Oil quantity & $\mathrm{Fe}$ & Grease & Oil quantity \\
\hline $22 \%$ & $12 \%$ & $78 \%$ & $22 \%$ & $12 \%$ & $78 \%$ \\
\hline
\end{tabular}

Table 2. Properties of the Lithium Grease

\begin{tabular}{ll}
\hline Properties & Lithium grease \\
\hline Dropping point ${ }^{\circ} \mathrm{C}$ & $177-204$ \\
Maximum usable temperature ${ }^{\circ} \mathrm{C}$ & 135 \\
Water resistance & Excellent \\
Oxidation stability & Excellent \\
Oil separation & Good to excellent \\
Appearance & Smooth and buttery \\
Protection against rust & Good \\
\hline
\end{tabular}

Table 3. Properties of the castor and honge oil

\begin{tabular}{llr}
\hline Properties & Castor oil & Honge oil \\
\hline Viscosity $(\mathrm{Pa}-\mathrm{s})$ & 0.03 & 0.048 \\
Flash point $\left({ }^{\circ} \mathrm{C}\right)$ & 270 & 230.000 \\
Fire point $\left({ }^{\circ} \mathrm{C}\right)$ & 310 & 282.000 \\
Specific gravity & 959 & 0.927 \\
Density $\left(\mathrm{kg} / \mathrm{m}^{3}\right)$ & 914 & 932.000 \\
Market cost (Rupees) & 80 & 120.000 \\
\hline
\end{tabular}

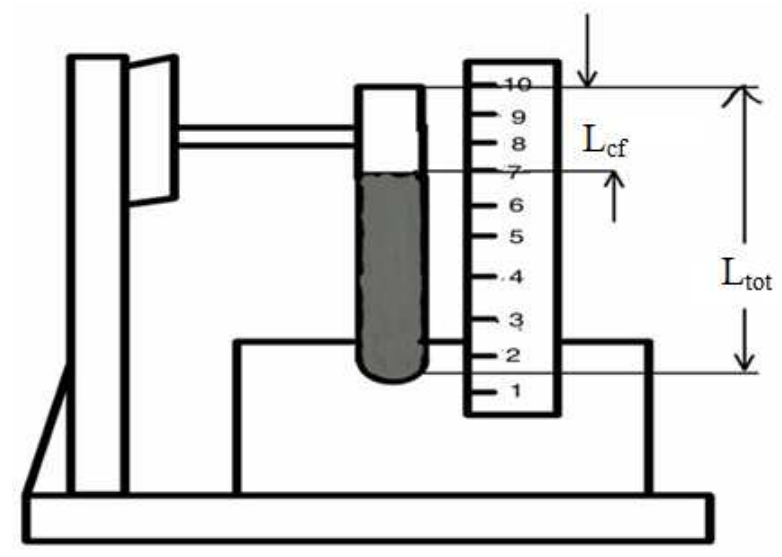

Fig. 1. Experimental setup for sedimentation

Let $\mathrm{L}_{\mathrm{cf}}$ be the length of the clear fluid

Let $\mathrm{L}_{\text {tot }}$ be the total length of prepared fluid, i.e., $10 \mathrm{~cm}$

From the graph Fig. 2a it can be observed that Honge oil sample has faster sedimentation ratio (180 h) in the beginning of experiment, leaving small amount of iron particles suspended in the supernatant fluid and then have lesser sedimentation ratio later on (after $180 \mathrm{~h}$ ). Same thing happens with the castor oil sample Fig. 2b, but here sedimentation occurs fast till $210 \mathrm{~h}$ and becomes almost constant after $210 \mathrm{~h}$. By comparing these two samples we can say that castor oil has good stability properties due to fast sedimentation of honge oil.

Figure 3 shows the relationship between the shear stress and strain. The Castor and Honge MR Fluid started to yield at a stress of 0.030 and $0.040 \mathrm{KPa}$. After the yield point, the shear stress increases gradually until it reaches a maximum 0.2 and $0.030 \mathrm{KPa}$ at a strain $5 \%$. With this comparison, the MR fluid became so stronger and much rigid. The elastic limit, the modulus and the yield stress were all increased dramatically. A large strain breaks the microstructure of MRF and leads to small increase of shear stress.

Figure 4 shows the experimental data for shear stress (Pa) at different shear rate $\left(\mathrm{s}^{-1}\right)$ for the 2 fluid samples. The graph Fig. 4 shows non-Newtonian fluid behavior for the MR Fluid samples in the absence of magnetic field and shear stress slightly increases with increase in shear rate. The graph also shows that the MR Fluid samples are following Bingham plastic model. At the higher shear rate both the fluid samples shows almost same behavior of shear stress.

Figure 5 shows the experimental data of dynamic viscosity $(\mathrm{Pa}-\mathrm{s})$ with respect to shear rate $\left(\mathrm{s}^{-1}\right)$ in the experimentation. We can see in the Fig. 5 that viscosity of both the MR Fluid samples decreases with increase in shear rate. With increase in shear rate the viscosity decreases and at high shear rate viscosity varies in both MR Fluid samples. A shear thinning behavior of the MR suspension can be observed.

Figure $6 a$ and $b$ shows the storage modulus $\left(G^{\prime}\right)$ and loss modulus $\left(\mathrm{G}^{\prime \prime}\right)$ as the function of angular frequency without magnetic field. Storage modulus gives an account of energy stored and recovered per cycle, known as storage modulus. The measure of energy dissipated or lost as heat per cycle is called as loss modulus.

The storage modulus is the measurement of the elasticity and loss modulus is the property related to the shear viscosity. Here by observing results we can say that, the samples posses superior solid kind structure rather than liquid like structure because both storage and loss modulus do not vary apparently with the increase in the frequency. It is also to be noted that the storage modulus is always higher than loss modulus over the frequency range. It is leading to the elastic property of the MR Fluids.

Figure $7 \mathrm{a}$ and $\mathrm{b}$ represents amplitude sweep curve. In the starting range of strain, the storage modulus shows a little dependence on the strain then declined gradually.

The frequency response of the loss factor $\left(G^{\prime \prime} / G^{\prime}\right)$ for both oil are illustrated in Fig. 8 it can be seen that loss factor is very sensitive to magnetic field.

The loss factors are not well behaved as the storage modulus and loss modulus: as driving frequency increases the loss factor increases gradually Fig. 8 by the way, the loss factor is also a function of the magnetic field.

Figure $9 \mathrm{a}$ and $\mathrm{b}$ shows the results of MR Fluids viscosity measured at room temperature $\left(27^{\circ} \mathrm{C}\right)$. By the Fig. $9 \mathrm{a}$ and $\mathrm{b}$ viscosity of both MR Fluids exhibits shear thinning behavior. 
Vikram G. Kamble et al. / American Journal of Nanotechnology 2015, 6 (1): 7.15

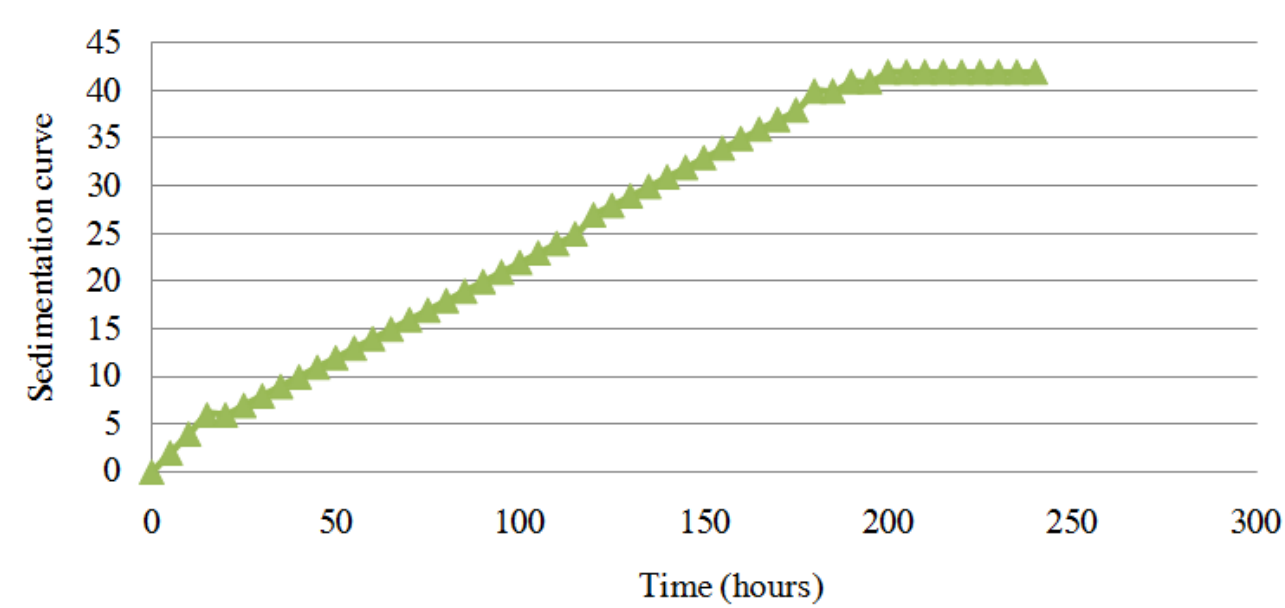

(a)

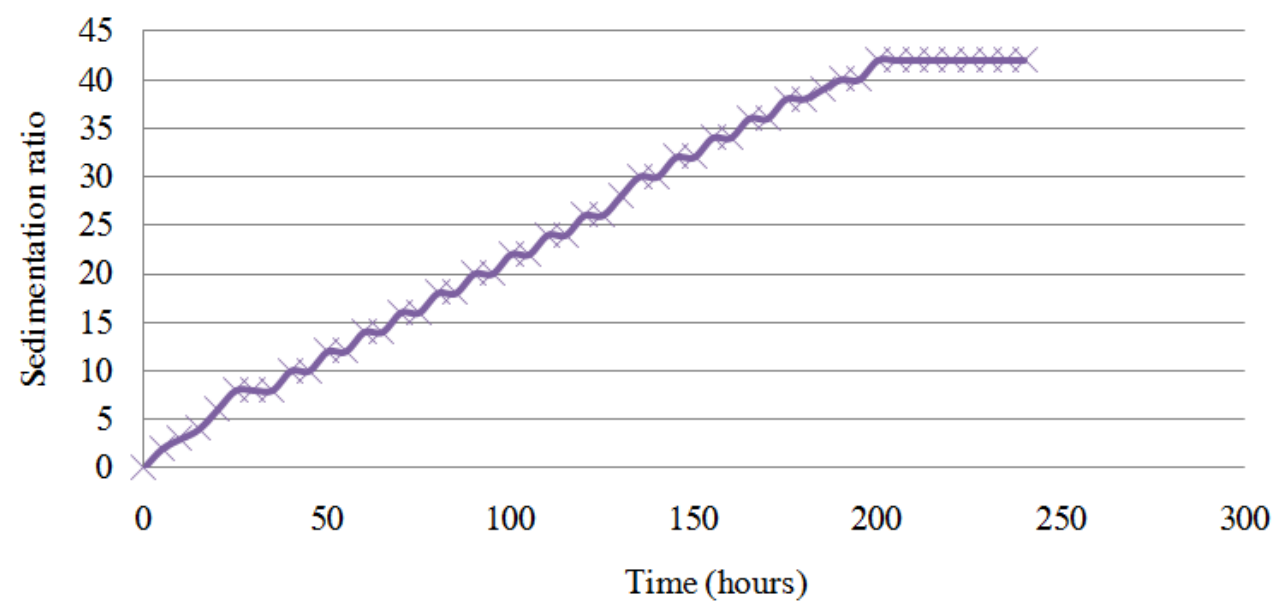

(b)

Fig. 2. (a) Sedimentation curve for honge oil (b) Sedimentation curve for castor oil

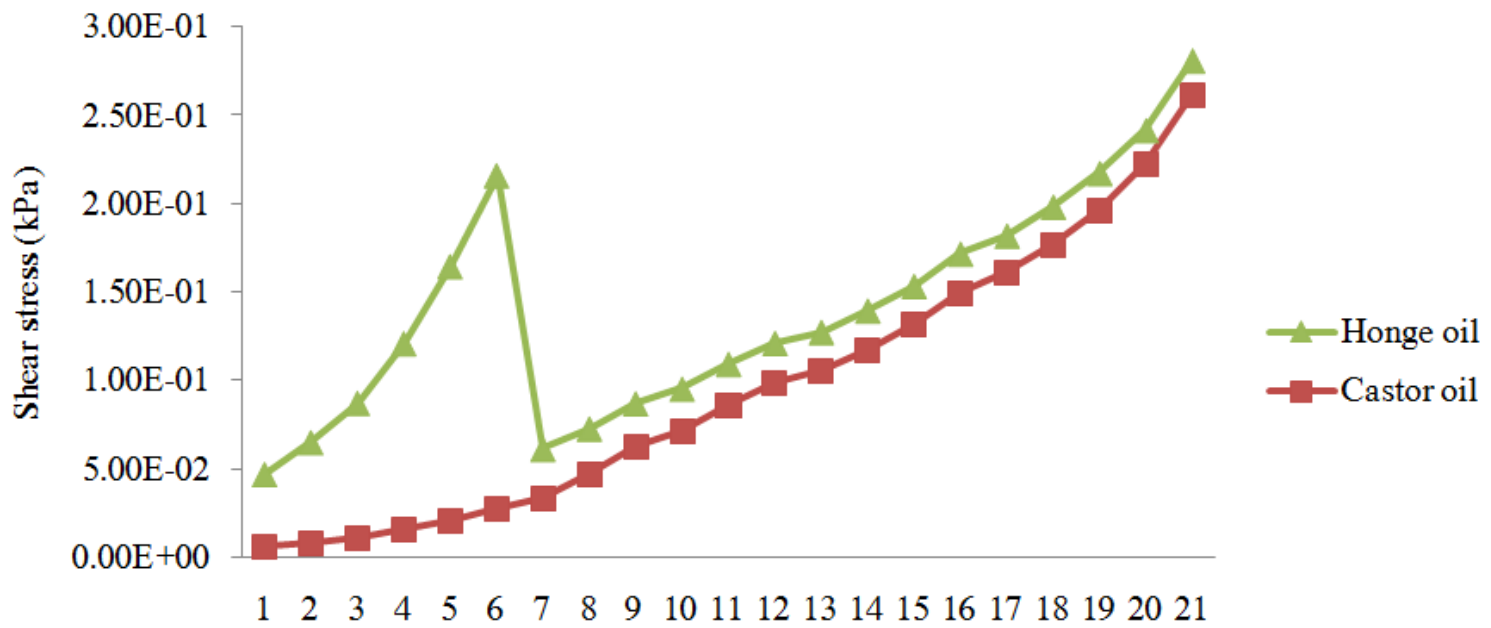

Shear strain $(\%)$

Fig 3. Shear stress as a function of Shear strain 


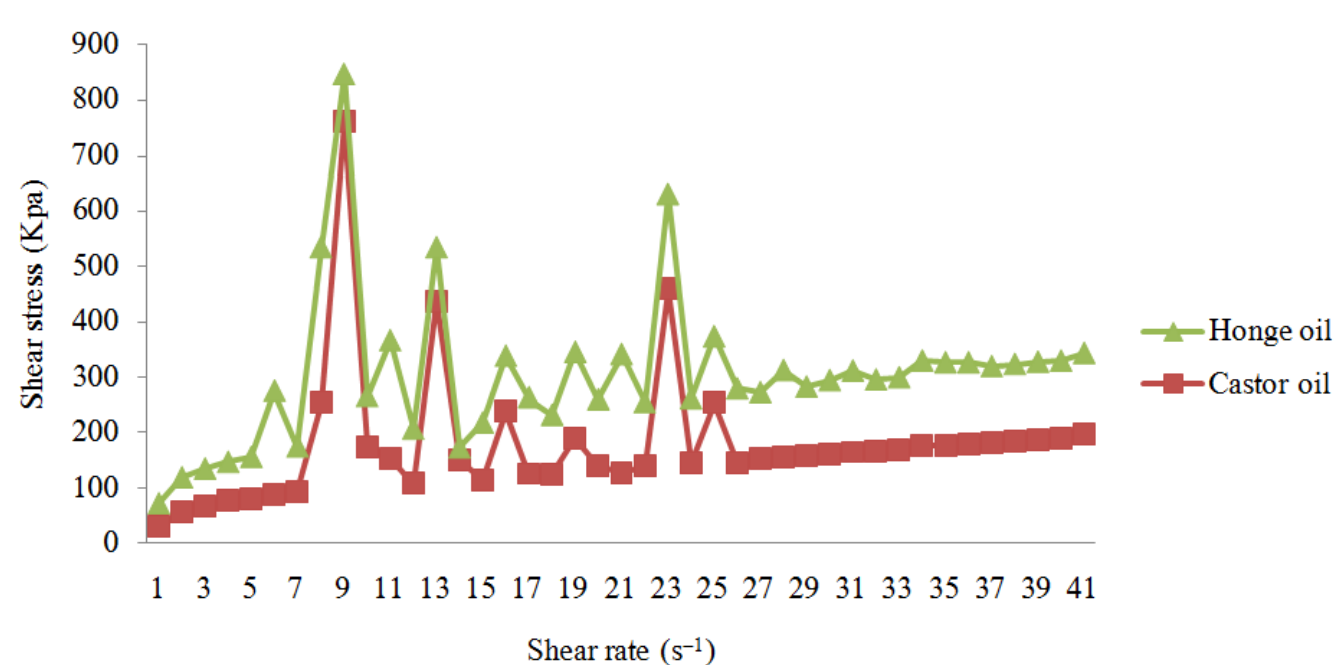

Fig. 4. Shear stress as a function of shear rate

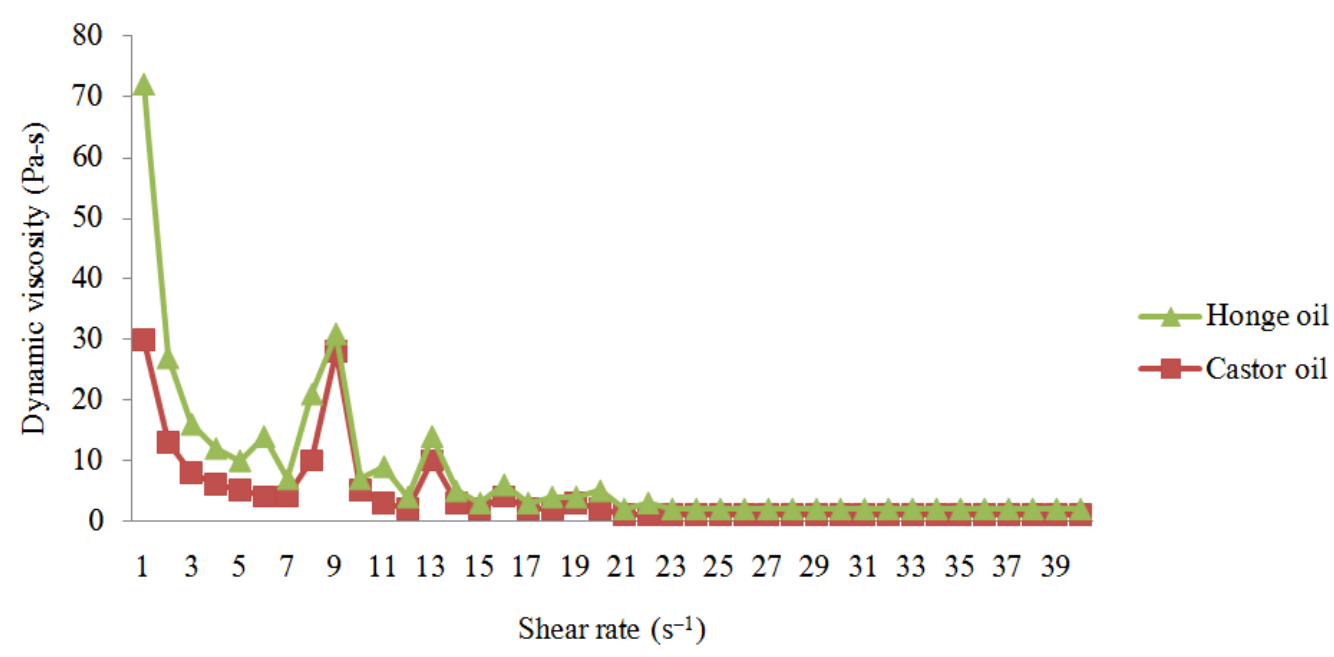

Fig. 5. Dynamic viscosity as a function of Shear rate

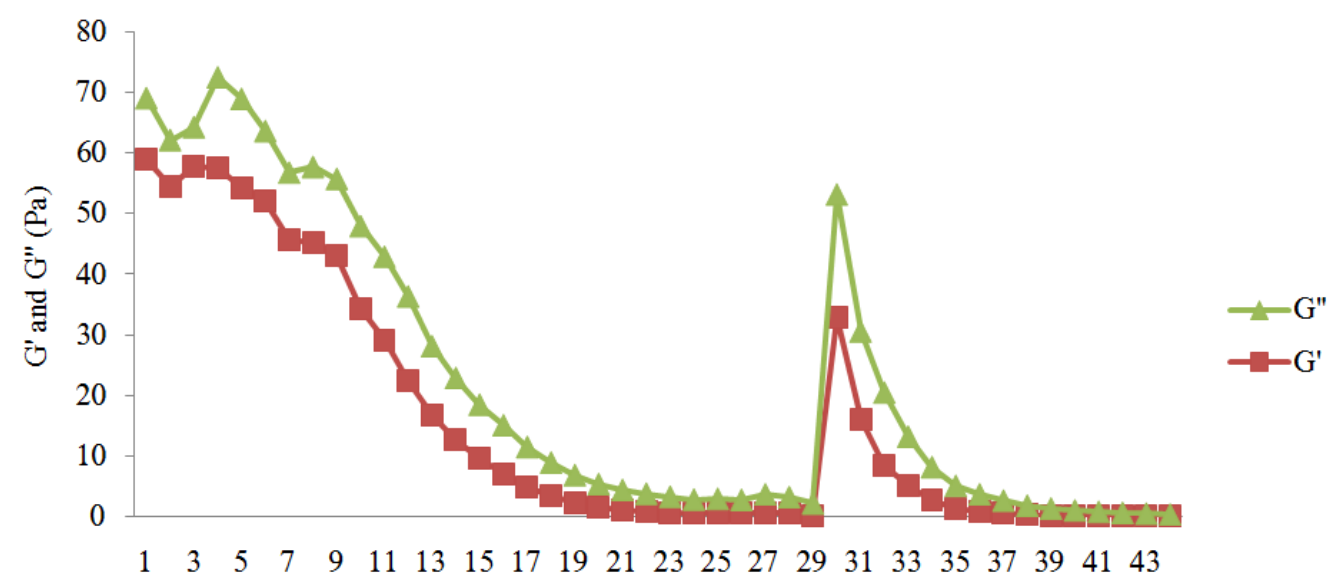

Angular frequency $(\mathrm{rad} / \mathrm{s})$

(a) 


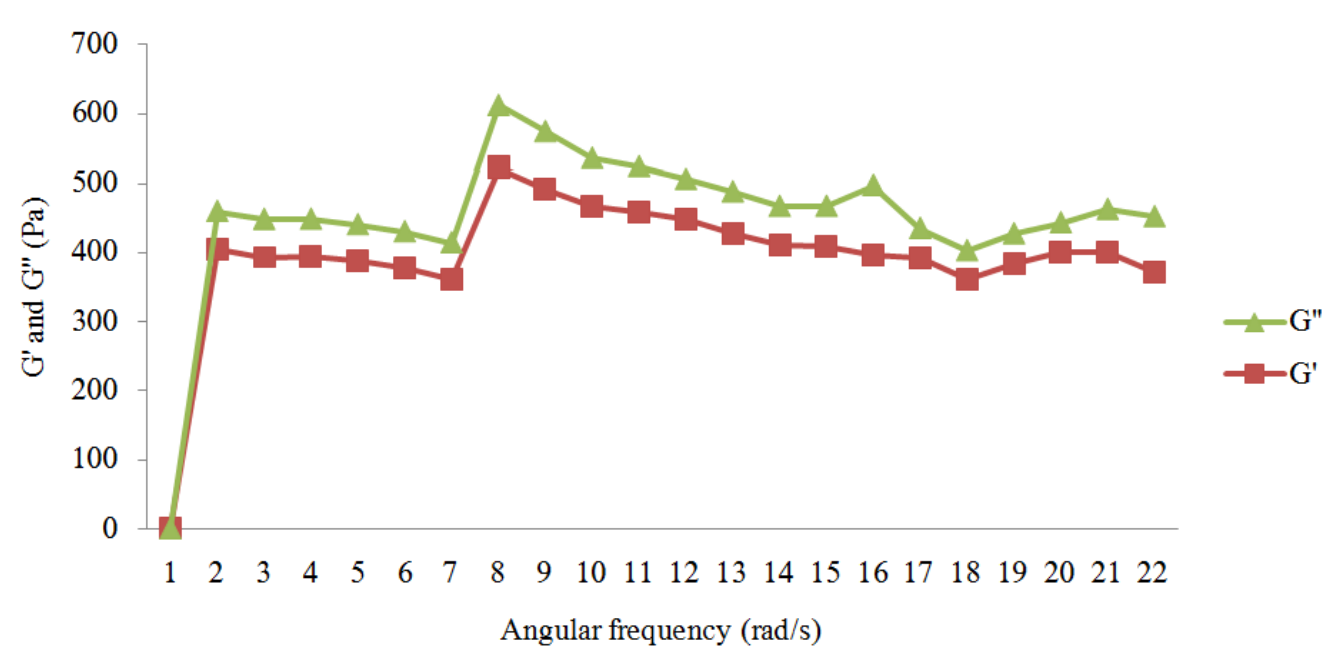

(b)

Fig. 6. (a) Storage and loss modulus as a function of angular frequency for Castor oil (b) Storage and loss modulus as a function of angular frequency for Honge oil

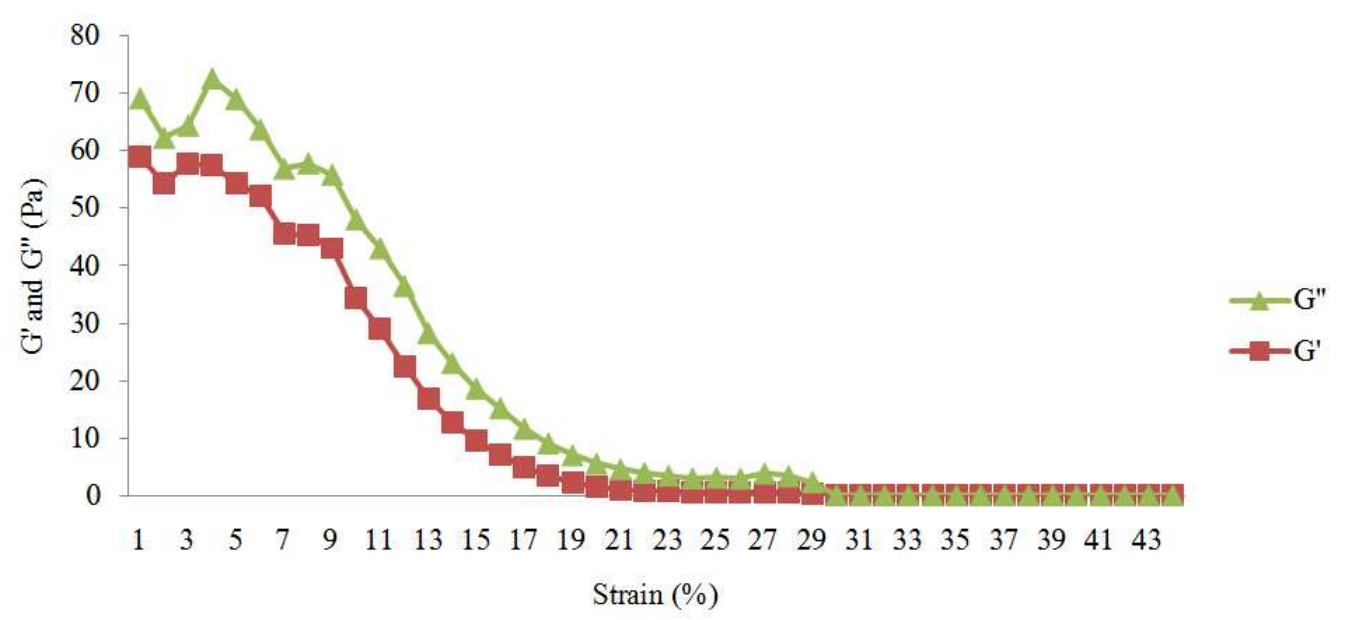

(a)

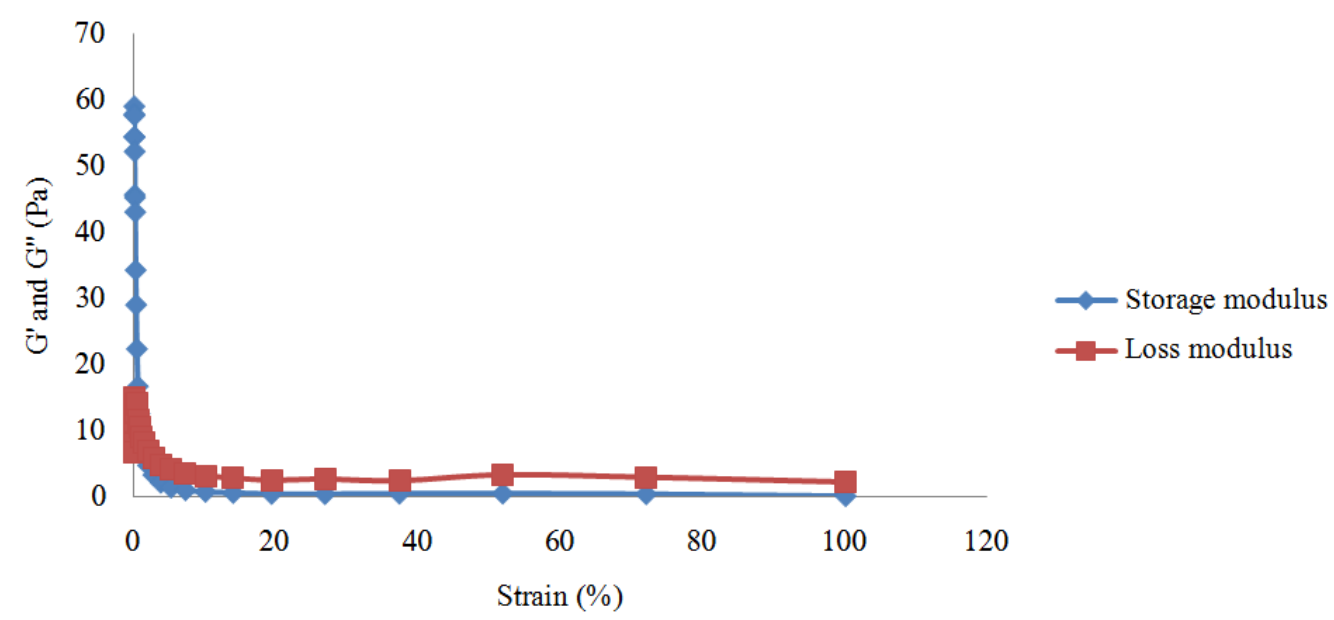

(b)

Fig. 7. (a) Storage and loss modulus as a function of Strain for Castor oil (b) Storage and loss modulus as a function of Strain for Honge oil 


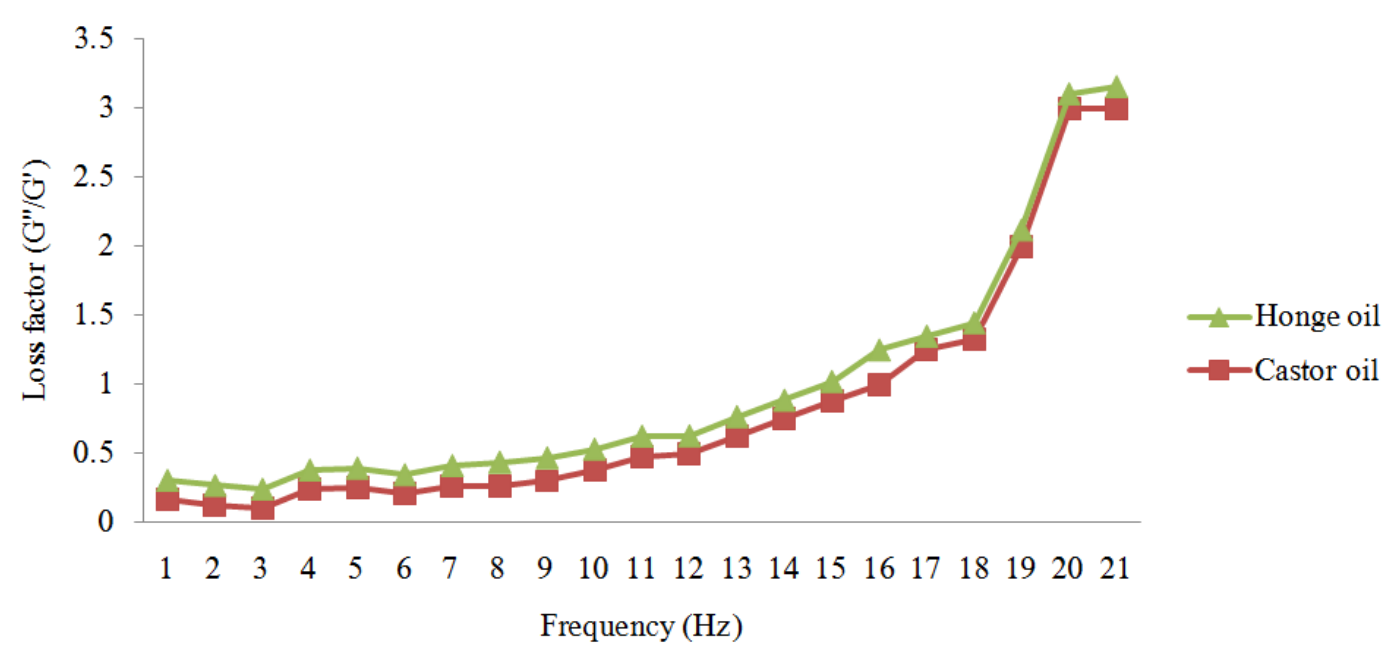

Fig. 8. Loss factor as a function of Frequency

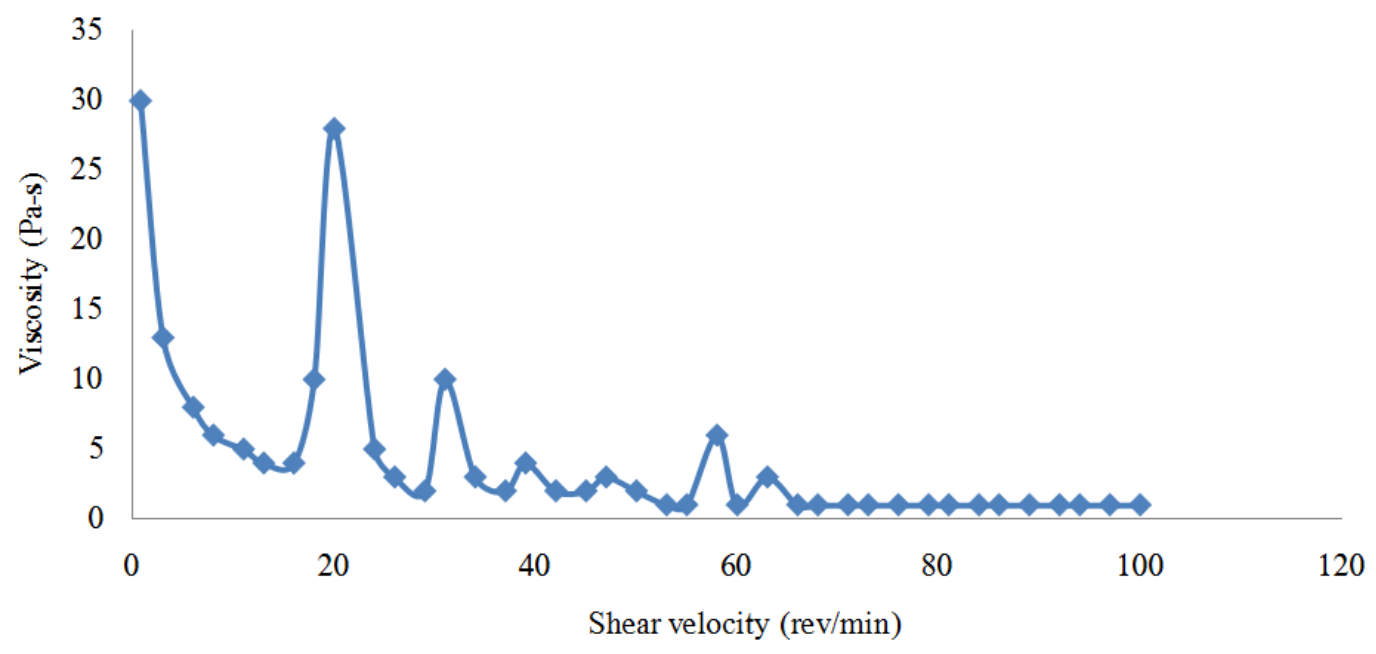

(a)

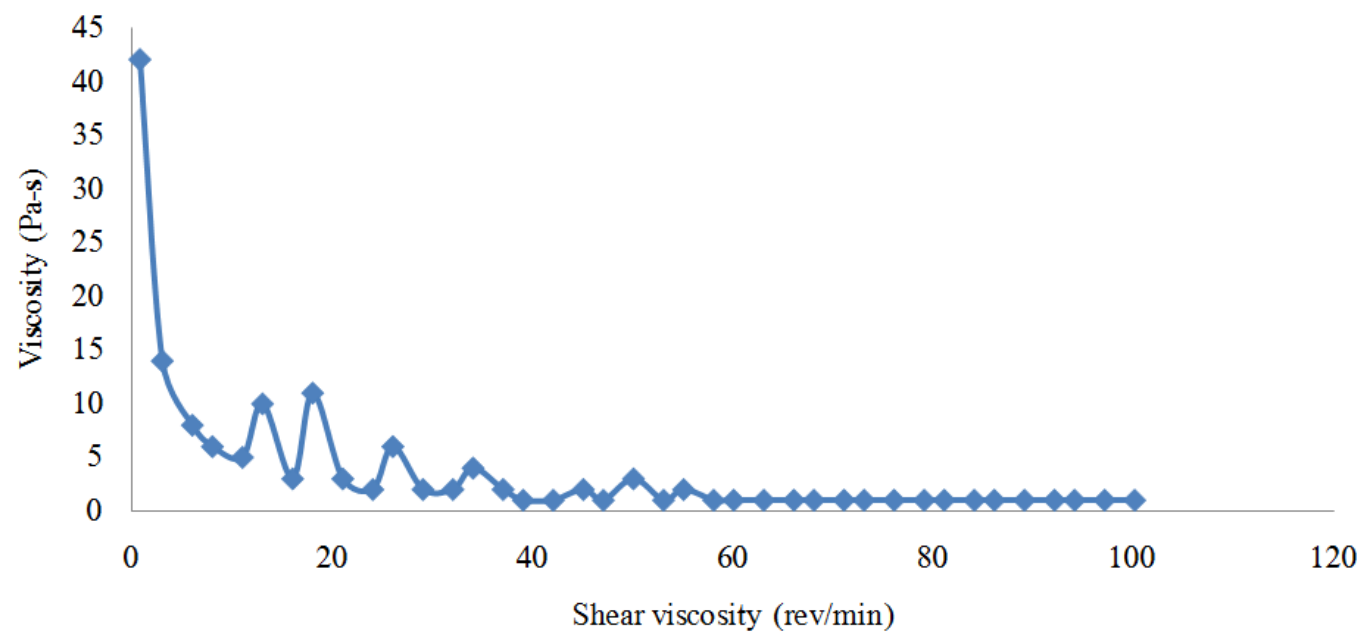

(b)

Fig. 9. (a) Viscosity as a function of Shear velocity of Castor oil (b) Viscosity as a function of Shear velocity of Honge oil 
Diameter of carbonyl iron particles has strong influence on the viscosity of MR Fluid-means that diameter of particles are almost in direct proportional to the viscosity of MF Fluid.

Comparing the viscosity of both castor and honge oil, it is easy to observe that the viscosity of honge oil is higher than that of castor oil.

\section{Conclusion}

In this study, the experimental investigation of MR fluids in terms of dynamic viscosity, frequency, storage modulus $\left(G^{\prime}\right)$, loss modulus $\left(G^{\prime \prime}\right)$ and loss factor $\left(G^{\prime \prime} / G^{\prime}\right)$ are presented. Results are summarized as follows:

- Storage modulus as well as loss modulus decreases with increase in strain percentage

- Storage modulus is always higher than the loss modulus over the frequency range and it is leading the elastic properties of MR samples

- Shear stress increases with increase in the shear rate. This property depicts that both samples are following Bingham plastic model

- With increase in shear rate the dynamic viscosity of samples decreases and we can observe the shear thinning behavior of the samples

\section{Acknowledgement}

We are gratefully acknowledge the support of the Malvern instruments New Delhi and St. Joseph Engineering College, Mangalore, Karnataka, India as they supported us in testing our samples and made our work successful.

\section{Author Contribution}

Vikram G Kamble: Participated in all experiments, coordinated the data-analysis, contributed to the writing of the manuscript and coordinated with mouse work.

Shreedhar Kolekar: Guided throughout the work.

Chetan Madivalar: Planned and organized the whole work.

\section{Ethics}

There will not be any ethical issues regarding the publication of this manuscript.

\section{References}

Boczkowska, A. and S. Awietjan, 2012. Microstructure and properties of magnetorheologicalelastomers. Licence Intech.

Boczkowska, A. and S.F. Awietjan, 2011. Effect of the elastomer stiffness and coupling agents on rheological properties of magnetorheologicalelastomers. Mater. Characterisation V. DOI: 10.2495/MC110231
Borcea, L. and O. Bruno, 2001. On the magneto-elastic properties of elastomer-ferromagnetcomposites. J. Mech. Phys. Solids, 49: 2877-2919. DOI: $10.1016 / \mathrm{S} 0022-5096(01) 00108-9$

Böse, H. and R. Röder, 2009. Magnetoreologicalelastomers with high variability of their mechanical properties. J. Phys. Conf. Ser., 149: 1-6. DOI: 10.1088/1742-6596/149/1/012090

Carlson, J.D. and M.R. Jolly, 2000. MR fluid, foam and elastomer devices. Mechatronics, 10: 555-569.

Chiriac, H. and G. Stoian, 2010. Influence of particle size distributions on magnetorheological fluid performance. J. Mater. DOI: $10.1088 / 1742-6596 / 200 / 7 / 072095$

Demchuk, S.A. and V.A. Kuzmin, 2002. Viscoelastic properties of magnetorheologicalelastomersin the regime of dynamic deformation. J. Eng. Physics Thermophysics, 75: 396-400. DOI: $10.1023 / \mathrm{A}: 1015697723112$

Dorfmann, A. and R.W. Ogden, 2003. Magnetoelastic modeling of elastomers. Eur. J. Mechanics A/Sol., 22: 497-507.

DOI: $10.1016 / \mathrm{S} 0997-7538(03) 00067-6$

Garg, D.P. and G.L. Anderson, 2000. Research in active composite materials and structures: An overview. Proc. SPIE, 3992: 2-12. DOI: 10.1117/12.388188

Ginder, J.M., S.M. Clark, W.F. Schlotter and M.E. Nichols, 2002. Magnetostrictive phenomena in magnetorheologicalelastomers. Int. J. Modern Phys. B, 16: 17-18.

Kamble, V.G. and S. Kolekar, 2014. Analysis of rheological properties of MR fluid based on variation in concentration of iron particles. Am. J. Nanotechnol., 5: 12-17. DOI: 10.3844/ajnsp.2014.12.16

Kamble, V.G. and V. Rvadi, 2015. Study of tool wear properties using magnetorheological fluid damper. Am. J. Nanotechnol.

Kankanala, S.V. and N. Triantafyllidis, 2004. On finitely strained magnetorheological elastomers. J. Mechanics Phys. Solids, 52: 2869-2908. DOI: 10.1016/j.jmps.2004.04.007

Kolekar, S., R.V. Kurahatti, P.K. Prashanth, V. Kamble and N. Reddy, 2014. Preparation of a silicon oil based magneto rheological fluid and an experimental study of its rheological properties using a plate and cone type Rheometer. J. ISSS, 3: 23-26.

Lokander, M., T. Reitberger and B. Stenberg, 2004. Oxidation of natural rubber-based magnetorheologicalelastomers. Polymer Degradation Stability, 86: 467-471. DOI: 10.1016/j.polymdegradstab.2004.05.019

Mangal, S.K., M. Kataria and A. Kumar, 2013. Synthesis of magneto rheological fluid. Int. J. Eng. Advanced Technol., 2: 20-25. 
Park, J.H., B.D. Chin and O.O. Park, 2001. Rheological properties and stabilization of magnetorheological fluids in a water-in-oil emulsion. J. Colloidal Interface Sci., 240: 349-354. DOI: $10.1006 /$ jcis. 2001.7622

Ray, S., M. Shanmugharaj and K. Bhowmick, 2002. A new parameter for interpretation of polymer-filler and filler-filler interactions in rubber vulcanizates. J. Mater. Sci. Lett., 21: 1097-1100.

DOI: $10.1023 / \mathrm{A}: 1016506532606$

Schwartz, M., 2002. Encyclopedia of Smart Materials vol. 1st Edn., Wiley, New York.

Takagi, T., 1999. Present state and future of the intelligent materials and systems in Japan. J. Intelligent Mater. Syst. Struct., 10: 575-581.

DOI: 10.1106/33UC-FBCW-342W-P2WW
Yalcintas, M. and H. Dai, 2004. Vibration suppression capabilities of magnetorheological materials based adaptive structures. Smart Mater. Struct.

DOI: 10.1088/0964-1726/13/1/001

Zhou, G.Y. and Z.J. Jiang, 2004. Deformation in magnetorheologicalelastomer and elastomerferromagnetcomposite driven by a magnetic field. Smart Mater. Struct., 13: 309-316. DOI: $10.1088 / 0964-1726 / 13 / 2 / 009$

Zhou, G.Y., 2003. Shear properties of magnetorheologicalelastomer. Smart Mater. Structures, 12: 139-146. 